\title{
General Psychiatry Postpartum psychosis in a non-native language-speaking patient: A perspective on language barriers and cultural competency
}

\author{
Tatsuhiko Naito (D) , ${ }^{1}$ Justin Chin (D) , ${ }^{1}$ Jun Lin (D) , ${ }^{1}$ Pritesh J Shah, ${ }^{2}$ \\ Christine M Lomiguen (i) ${ }^{3}$
}

To cite: Naito T, Chin J, Lin J, et al. Postpartum psychosis in a non-native language-speaking patient: A perspective on language barriers and cultural competency. General Psychiatry 2019;32:e100077. doi:10.1136/ gpsych-2019-100077

Received 09 April 2019 Revised 05 May 2019 Accepted 15 May 2019
Check for updates

(C) Author(s) (or their employer(s)) 2018. Re-use permitted under CC BY-NC. No commercial re-use. See rights and permissions. Published by BMJ.

${ }^{1}$ Primary Care, Touro College of Osteopathic Medicine, New York City, New York, USA

${ }^{2}$ Psychiatry, Holy Name Medical Center, Teaneck, New Jersey, USA

${ }^{3}$ Basic Sciences, Lake Erie College of Osteopathic Medicine, Erie, Pennslyvannia, USA

Correspondence to Mr Tatsuhiko Naito; tnaito2@student.touro.edu

\section{SUMMARY}

Postpartum psychosis is a condition characterised by rapid onset of psychotic symptoms several weeks after childbirth. Outside of its timing and descriptions of psychotic features, minimal research exists due to its relative rarity ( 1 to 2 per 1000 births in the USA), with greater emphasis on postpartum sadness and depression. With the existing literature, cultural differences and language barriers previously have not been taken into consideration as there are no documented cases of postpartum psychosis in a non-English-speaking patient. Correctly differentiating postpartum psychosis from other postpartum psychiatric disorders requires adeptly evaluating for the presence of psychotic symptoms with in-depth history taking. Here, we present a case of postpartum psychosis in a Japanese-speaking only patient, with an associated clinical course and culturally appropriate approach to treatment. A review of postpartum psychosis and language/cultural considerations are also discussed, with attention on the Japanese concept of 'Satogaeri bunben'.

\section{INTRODUCTION}

Postpartum psychosis (PP) is a condition characterised by rapid onset of psychotic symptoms several weeks after childbirth. It is a psychiatric emergency that requires immediate detection and intervention due to the gravity of its consequences, ranging from suicide to infanticide. ${ }^{1}$ Hence, better understanding of risk factors is paramount in facilitating timely response and early intervention. Risk factors associated with PP; however, are poorly understood due to its relative rarity (1-2 out of 1000 childbearing women). ${ }^{12}$ While multiple studies have elucidated several epidemiological risk factors, including, but not limited to, history of bipolar disorder and primiparity, very little research explores sociodemographics, such as ethnicity or cultural practices, let alone one particular population. ${ }^{1-5}$ With no reports regarding PP among Japanese women in the USA, the aim of this case report is to present potential risk factors and management challenges unique to the population.

\section{CASE PRESENTATION}

Ms. $\mathrm{K}$ is a 35-year-old Japanese woman with a medical history of hypothyroidism brought to the emergency department (ED) after she attempted to stab herself with a knife. Her husband was able to interfere before the act was completed. In the ED, she presented with a depressed mood and expressed suicidal thoughts. One week prior to the incident, she had an uncomplicated vaginal birth to a baby boy at the same hospital and had no prenatal health issues. Several days after her discharge, she experienced multiple episodes of diaphoresis and became disoriented to time and place, which led to her current presentation. Of note, Ms. K had been living in the USA for only 5 months and spoke very limited English; her husband contributed the majority of the history.

Initial physical examination showed an alert, adequately nourished, anxious woman. Vital signs were within normal limits, except for an elevated blood pressure of 159/91. Head and neck, cardiovascular, respiratory, gastrointestinal, neurological and dermatological examinations were benign. During mental status examination, her affect was incongruent to her mood in which she would be smiling and cheerful, despite stating that she was anxious and sad. Her mood was labile, ranging from calm cooperation to extreme agitation. She expressed helplessness but not hopelessness and denied suicidal or homicidal ideation, intent or plans. Her insight, judgement and cognition appeared to be impaired, with repetitive speech and occasional lapses in concentration and focus but no extrapyramidal symptoms 
or abnormal involuntary muscle movements. Although she initially did not display any overt sign of hallucinations, she exhibited indications of delusion as she continuously expressed deep suspicion towards the off-site telephone translation service used during intake. She was unable to see the interpreter and repeatedly stated that the machine was trying to trick her. Because of this, her clinical picture was further complicated as communication was limited to translation by her husband and was inconclusive in deciphering her symptoms.

She was admitted to the inpatient psychiatric unit for observation and concerns of safety for her baby and self. Complete blood count, complete metabolic panel, thyroid panel, cholesterol panel, urine toxicology and culture were unremarkable. On hospital day 1 , she showed signs of derealisation in which she admitted that she felt as if she were in a "dreamlike state" and that her attempt to self-harm was "just things happening in my dream". She also showed a sign of disorientation as she stated she felt her "internal clock was broken and still stuck at the point she gave birth to her baby". Since moving to the USA, she has had minimal human interaction other than her husband who has a 14-hour work day. Her internet-based communication with her siblings and friends in Japan was limited due to the time difference. She stated that in addition to the isolation, she felt overwhelmed in taking care of her baby while also trying to minimise her negative feelings so that she would not be a burden to her husband. She felt guilty, but relieved as she said her parents were coming to the USA after hearing of her hospitalisation. She later confided to staff that she had a psychiatric history of depressive symptoms related to stress from her work 13 years ago for which she saw a psychiatrist but was not formally diagnosed and declined pharmaceutical intervention at that time. She denied any family history of psychiatric history and stated that her husband and family were not aware of her psychiatric history.

With the language barrier contributing to the lack of clear, definitive signs for PP, Ms. K was initially started on sertraline (Zoloft) $50 \mathrm{mg}$ (increased to $75 \mathrm{mg}$ the following day), lorazepam (Ativan) $0.5 \mathrm{mg}$ and zolpidem (Ambien) $5 \mathrm{mg}$, with the instructions for staff to monitor for signs of overt psychosis. Levothyroxine $75 \mu \mathrm{g}$ daily was continued for hypothyroidism. Minimal improvement occurred from hospital days 1 to 3 and her family was advised that she would need to stay for several days to ensure that her symptoms improve and that she would not be a danger to herself or others. On hospital day 3 , she suddenly admitted to an on-site Japanese-speaking staff member that she was hearing indistinct voices, in which the attending was contacted and aripriprazole (Abilify) $5 \mathrm{mg}$ was immediately started. Her mood and negative symptoms rapidly stabilised over the following days, which ultimately confirmed diagnosis of postpartum psychosis. She was discharged on hospital day 6 with outpatient follow-up with Japanese-speaking psychiatrists and therapists.

\section{DISCUSSION}

$\mathrm{PP}$ is relatively a rare disorder that most commonly presents within the first 2-4 weeks after delivery, but can emerge as early as 2-3 days after childbirth. ${ }^{12}$ Rapid mood fluctuation is considered the hallmark; however, the rapid onset of paranoia, grandiosity, bizarre delusions, confused thinking and grossly disorganised behaviour may also be present. ${ }^{13}$ Insomnia, anxiety or depressed mood have been reported as early warning symptoms prior to PP. ${ }^{4} \mathrm{PP}$ is a unique disease in that its trigger is definable: childbirth. Exact pathophysiology of the disease; however, has yet to be determined, with several theories ranging from hormonal change to immune dysregulation and circadian rhythm disruption. ${ }^{34}$ In this particular case, the patient's history of hypothyroidism and levothyroxine use during pregnancy gives credence to an autoimmune aetiology to her PP, with literature demonstrating a strong association between PP and autoimmune thyroiditis/thyroid dysfunction. ${ }^{67}$ Understanding of risk factors would facilitate timely and appropriate response from health professionals via heightened awareness and proper preventive measures such as lithium prophylaxis. These risk factors related to PP; however, are still poorly understood and rarely studied.

$\mathrm{PP}$ is a clinical diagnosis. The DSM-5 does not recognise $\mathrm{PP}$ as a distinct condition; rather, it adds a "with a postpartum onset" specifier if a woman met criteria for a brief psychotic disorder during or within 4 weeks post partum. ${ }^{2}$ Due to its similar presentation, bipolar disorder is an important differential diagnosis that must be considered, especially with prior history. ${ }^{3}$ Other psychiatric conditions that must be included, but are not limited to, are generalised anxiety disorder, obsessive compulsive disorder and postpartum depression, as well as organic causes of psychosis such as infection, recreational drugs and electrolyte disturbances to name a few. ${ }^{3}{ }^{7}$ Notwithstanding the classification in DSM-5, PP is a psychiatric emergency that requires urgent evaluation, psychiatric referral and inpatient hospitalisation due to the gravity of consequences, such as suicide, infanticide, impaired motherinfant bonding, infant abuse and neglect. ${ }^{16}$ While the overall prognosis is positive for women who have sought help, screening protocols or treatment guidelines have yet to be established. ${ }^{14}$ Atypical antipsychotics, mood stabilisers and antiepileptics are common drugs of choice along with electroconvulsive therapy; however, there is a paucity of evidence to support their efficacy due to the difficulty of randomised trials. ${ }^{134}$

In examining Ms. K, she represented several potential risk factors of PP that are unique to her experience as a non-English-speaking Japanese immigrant, which warrants further discussion. With consideration of the sociodemographic factors of her situation-her husband working long hours, being separated from her family and friends, inability to speak English and unfamiliarity with her new environment-it was evident that the patient severely lacked proper support in the perinatal period. Lack of social support has been strongly associated to 


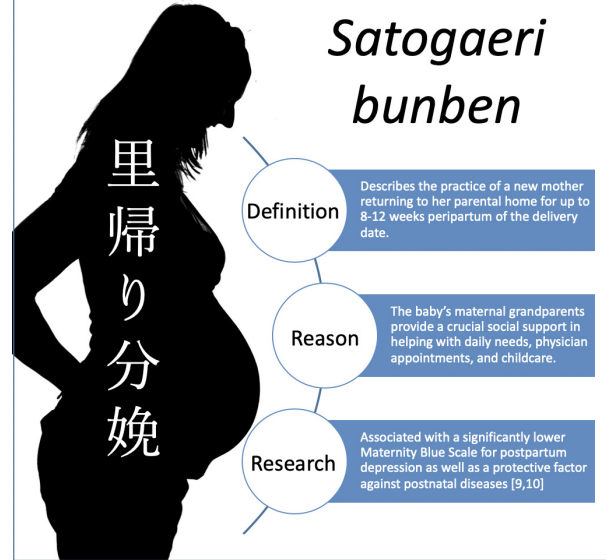

Figure 1 Infographic for 'Satogaeri bunben' in describing its definition, rationale and associated research.

postpartum depression. ${ }^{5}$ More specifically, the absence of the husband/partner during the perinatal and peripartum period has been shown to increase rates of PP in other cultures. ${ }^{58}$ Inability to communicate with her physical surrounding, which in this case was manifested by a lack of English proficiency, is common among recent immigrants where English is not a primary language. The lack of English fluency in the USA and other countries where English is the primary language complicates PP identification and treatment as diagnosis requires extensive history taking. ${ }^{3}$ Furthermore, psychoeducational support is considered a crucial adjunct to pharmacological treatment following the discharge; lack of English fluency can significantly limit available resources. ${ }^{134}$

Unique to this case is the Japanese custom of Satogaeri bunben, which describes the practice of the new mother returning to her parental home for up to 8-12 weeks peripartum of the delivery date (figure 1). During this time, the baby's maternal grandparents provide a crucial social support in helping with daily needs, physician appointments and childcare after birth. ${ }^{9}$ Although there has been no empirical research in analysing a direct association between Satogaeri bunben and PP, several studies have demonstrated that the practice is associated with a significantly lower Maternity Blue Scale for postpartum depression as well as a protective factor against postnatal diseases. ${ }^{9}{ }^{10}$ Interestingly enough, Ms. K's situation could not have been more different from that of Satogaeri bunben: she was in an extremely unfamiliar and stressful environment where she could barely communicate with others around her and had no support of her parents during the perinatal period. Due to its cost, distance and reluctance to leave their husbands alone, it is increasingly more difficult for Japanese women in the USA to practise Satogaeri bunben, which makes it another potential risk factor applicable to this population.

\section{CONCLUSION}

Japanese-born women living in the USA face unique circumstances that render them more vulnerable to PP and make its management more challenging. While greater research is needed to substantiate some of these potential risk factors, it has several implications. Once established, the vulnerable population can be educated of the risk factors at the community level so they can consciously engage in non-medical protective measures, such as Satogaeri bunben or more active attempts to build supportive groups. It would also lead to better detection of early signs of PP by family members which would greatly decrease the likelihood of consequences via early professional intervention. Cultural competency and awareness are needed for physicians and healthcare professionals who work with immigrant/non-native communities. Finally, this case is a stark reminder that different cultures can be predisposed to certain psychiatric disorders in unique ways such that research and management plans must be catered accordingly.

Acknowledgements The authors would like to acknowledge the psychiatric team at Holy Name Medical Center, in particular, nurse manager Denielle Giuliani, for their assistance in this endeavour.

Contributors TN: data collection, literature review, developed the manuscript, edited the manuscript. JC: concept development, developed the manuscript, edited the manuscript. JL: data collection, developed the manuscript, edited the manuscript. PJS: data collection, edited the manuscript. CML: concept development, developed and edited the manuscript.

Funding The authors have not declared a specific grant for this research from any funding agency in the public, commercial or not-for-profit sectors.

Competing interests None declared.

Patient consent for publication Obtained.

Provenance and peer review Not commissioned; externally peer reviewed.

Open access This is an open access article distributed in accordance with the Creative Commons Attribution Non Commercial (CC BY-NC 4.0) license, which permits others to distribute, remix, adapt, build upon this work non-commercially, and license their derivative works on different terms, provided the original work is properly cited, appropriate credit is given, any changes made indicated, and the use is non-commercial. See: http://creativecommons.org/licenses/by-nc/4.0/.

ORCID IDs

Tatsuhiko Naito http://orcid.org/0000-0003-0872-0259

Justin Chin http://orcid.org/0000-0002-3540-4596

Jun Lin http://orcid.org/0000-0001-6820-5246

Christine M Lomiguen http://orcid.org/0000-0001-6964-6289

\section{REFERENCES}

1 Sit D, Rothschild AJ, Wisner KL. A review of postpartum psychosis. J Womens Health 2006;15:352-68.

2 VanderKruik R, Barreix M, Chou D, et al. The global prevalence of postpartum psychosis: a systematic review. BMC Psychiatry 2017;17.

3 Bergink V, Rasgon N, Wisner KL. Postpartum psychosis: madness, mania, and melancholia in motherhood. Am J Psychiatry 2016;173:1179-88.

4 Osborne LM. Recognizing and managing postpartum psychosis: a clinical guide for obstetric providers. Obstet Gynecol Clin North Am 2018;45:455-68.

5 Upadhyaya S, Sharma A, Raval C. Postpartum psychosis: risk factors identification. N Am J Med Sci 2014;6:35-7.

6 Bergink V, Kushner SA, Pop V, et al. Prevalence of autoimmune thyroid dysfunction in postpartum psychosis. Br J Psychiatry 2011;198:264-8.

7 Spinelli MG. Postpartum psychosis: detection of risk and management. Am J Psychiatry 2009;166:405-8.

8 Nager A, Johansson L-M, Sundquist K. Are sociodemographic factors and year of delivery associated with hospital admission for 
postpartum psychosis? A study of 500,000 first-time mothers. Acta Psychiatr Scand 2005;112:47-53.

9 Okano T, Nomura J, Kumar R, et al. An epidemiological and clinical investigation of postpartum psychiatric illness in Japanese mothers. J Affect Disord 1998;48:233-40.
10 Takahashi Y, Tamakoshi K. Factors associated with early postpartum maternity blues and depression tendency among Japanese mothers with full-term healthy infants. Nagoya J Med Sci 2014;76:129-38.

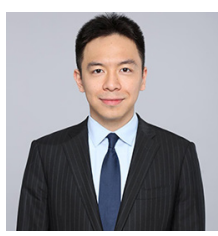

Tatsuhiko Naito is a third-year medical student at Touro College of Medicine in New York. Originally from Japan, he completed his undergraduate degree at Johns Hopkins University as a double major in Biology and Psychology. Subsequently he received his master degree in Medical Science from Boston University School of Medicine. During his undergraduate medical education career, he has served as the chapter president of the psychiatry interest group and student representative for his school's wellness committee. His psychiatry research interests range broadly from the utility of mindfulness to the application of culturally sensitive practices in medicine. 